DOI: 10.36910/6775-2524-0560-2020-39-18

УДК: 539.1.074+539.2

Федотов Вячеслав Віталійович, ст.викладач

https://orcid.org/0000-0001-7821-0989

Національний технічний університет України «Київський політехнічний інститут імені Ігоря Сікорського», м. Київ, Україна

\title{
МАТЕМАТИЧНА МОДЕЛЬ ЗБУДЖЕННЯ СПІНОВОГО ФОТОСТРУМУ У НАНОСТРУКТУРАХ АНТИФЕРОМАГНІТНИХ ДІЕЛЕКТРИКІВ
}

\begin{abstract}
Федотов В. В. Математична модель збудження спінового фотоструму у наноструктурах антиферомагнітних діелектриків. Розглянуто фізичні принципи, що лежать у основі фотогальванічного ефекту, який виникає внаслідок проходження циркулярно поляризованого світла у середовищі металу і виражається у генеруванні прямого фотоструму, напрям якого залежить від поляризації світла. Визначено особливості виникнення даного ефекту у структурах антиферомагнітних діелектриків, зазначено його нелінійність та запропоновано подальше використання у опто-спінтронних запам’ятовуючих пристроях і нанодетекторах електромагнітного поля. У рамках побудови математичного апарату розгляд спінового імпульсу було перенесено 3 фотонів на магнони. Таким чином, було вказано, що циркулярно поляризоване електромагнітне поле в рамках моделі генерує прямий спіновий фотострум магнона, причому напрям струму визначається спіральністю світла. Показано, що явище резонансу на базі загальних принципів нанофотоніки може бути представлено як взаємодія другого порядку поляризованого світла з речовиною. Розроблена математична модель була вдосконалена через врахування внеску геометричної організації складних гратчастих структур антиферомагнітних діелектриків і явище антисиметричного обміну.

Ключові слова: наноструктури антиферомагнітних діелектриків, круговий фотогальванічний ефект, магнонний спіновий фотострум, циркулярно поляризоване світло, взаємодія другого порядку світла 3 речовиною, антисиметричний обмін, складні гратчасті структури.
\end{abstract}

Федотов В. В. Математическая модель возбуждения спинового фототока в наноструктурах антиферромагнитных диэлектриков. Рассмотрены физические принципы, лежащие в основе фотогальванического эффекта, который возникает в результате прохождения циркулярно поляризованного света в среде металла и выражается в генерировании прямого фототока, направление которого зависит от поляризации света. Определены особенности возникновения данного эффекта в структурах антиферромагнитных диэлектриков, указано его нелинейность и предложено дальнейшее использование в опто-спинтронных запоминающих устройствах и нанодетекторах электромагнитного поля. В рамках построения математического аппарата рассмотрение спинового импульса было перенесено из фотонов на магноны. Таким образом, было указано, что циркулярно поляризованное электромагнитное поле в рамках модели генерирует прямой спиновый фототок магнонов, причем направление тока определяется спиральностью света. Показано, что явление резонанса на базе общих принципов нанофотоники может быть представлено как взаимодействие второго порядка поляризованного света с веществом. Разработанная математическая модель была усовершенствована через учет геометрической организации сложных решетчатых структур антиферромагнитных диэлектриков и явление антисимметричного обмена.

Ключевые слова: наноструктуры антиферромагнитных диэлектриков, круговой фотогальванический эффект, магнонный спиновый фототок, циркулярно поляризованный свет, взаимодействие второго порядка света с веществом, антисимметричный обмен, сложные решетчатые структуры.

Fedotov V. V. Mathematical model of spin photocurrent excitation in of antiferromagnetic isolators nanostructures

Fundamental principles of circular photogalvanic effect as a result of circularly polarized light probing of metal environment and generation of a direct photocurrent which direction depends on the polarization of light are considered. The peculiarities of the occurrence of the mentioned effect in the structures of antiferromagnetic dielectrics are determined, nonlinearity of the effect is indicated, and further use in opto-spintronics' data storage and nanodetectors of the electromagnetic field is proposed. Due to necessity of the mathematical apparatus development, the consideration of the spin pulse was transferred from photons to magnons. Thus, it was stated that the circularly polarized electromagnetic field within the model generates a direct magnon spin photocurrent and the direction of the current is determined by the helicity of light. It is shown that the phenomenon of resonance based on general principles of nanophotonics can be represented as the interaction of the second order polarized light-matter interaction. The mathematical model was further adopted in order to take into account the contribution of the geometric organization of antiferromagnetic isolator complex lattice structures and the phenomenon of antisymmetric exchange.

Keywords: antiferromagnetic isolators nanostructures, circular photogalvanic effect, magnon spin photocurrents, circularly polarized light, second order light-matter interaction, antisymmetric exchange, complex lattice structures.

Постановка наукової проблеми. На сьогоднішній день структури на основі антиферомагнітних діелектриків розглядаються як один 3 найбільш перспективних матеріалів спінтроніки $[1,2]$. Математичні моделі, що визначають процеси генерації та циркуляції спінових струмів електронів описуються через такі квазічастинки як магнони [10, 11]. Типові властивості антиферомагнітних магнонів вказують на широку сферу застосувань наноструктур антиферомагнітних діелектриків, зокрема пропонується використовувати даного підходу при побудові спінтронних запам'ятовуючих пристроїв, що характеризуються високою інформаційною ємністю та високоефективних детекторів електромагнітного поля, що визначає рівень актуальності даного дослідження. 
Аналіз сучасних досліджень та публікацій у зазначеній галузі вказує на перспективність застосування механізму ультрашвидкого оптичного збудження магнонів у структурах на основі антиферомагнітних діелектриків [1, 2], що дозволяє виділити окрему галузь антиферомагнітної оптоспінтроніки. 3 метою побудови математичної моделі відповідного процесу було досліджено такі явища нанофотоніки як хіральність і суперхіральність фотонів $[3-5,7,8]$, принципи взаємодії світла 3 речовиною $[6,9]$ та особливості збудження магнонного спінового фотоструму у антиферомагнетиках $[10,11,20,21]$. Також для уточнення математичного апарату, що описує механізми збудження спінових струмів у феромагнетиках та антиферомагнетиках було використано роботи по моделюванню у галузі квантової теорії магнетизму [12-19], зокрема математичні моделі, що враховують внесок геометричної організації складних гратчастих структур феромагнетиків та антиферомагнетиків [21-23] та явище антисиметричного обміну [24].

Зазначені матеріали наукових публікацій, тим не менш, не надають цілісної методології математичного моделювання та розробки опто-спінтронних пристроїв на базі антиферомагнітних наноструктур, що може бути виділено як невирішену частину загального дослідження. Таким чином, за мету даного дослідження може бути поставлено задачу побудови методологічної бази для аналізу процесів генерації та циркуляції спінових магнонних струмів у відповідності до структурних особливостей середовища антиферомагнітних діелектриків.

Виклад основного матеріалу й обгрунтування отриманих результатів дослідження.

1. Збудження фотоструму у середовищі провідника та діелектрику

Моделювання механізму генерації спінового струму у середовищі металу або антиферомагнітного діелектрику базується на врахування пучка поляризованого світла яким опромінюється зразок та фотогальванічному ефекті(рис. 1, 2).

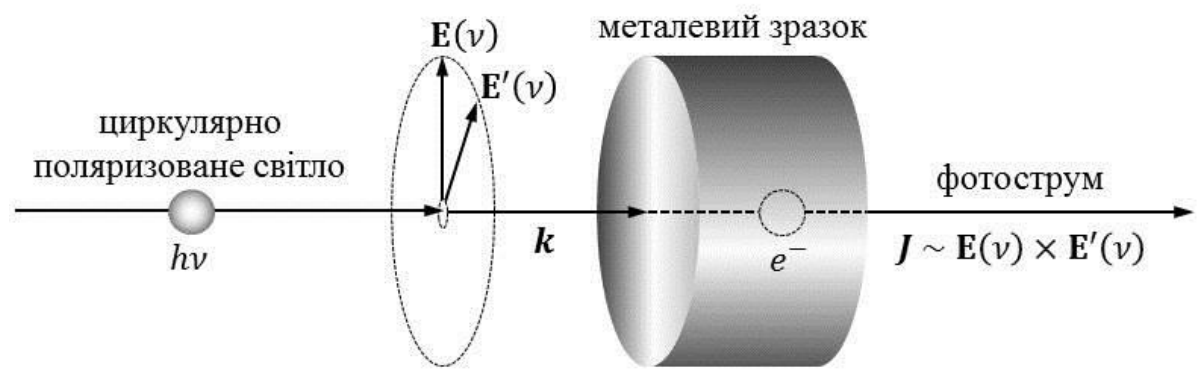

Рис. 1. Базова модель генерації фотоструму цииркулярно поляризованим світлом у металевому зразку

У середовищі металу круговий фотогальванічний ефект (circular photogalvanic effect, CPGE)слід розглядати як нелінійний процес, що відображає взаємодію зразка і електромагнітного поля циркулярно поляризованої хвилі, напруженість якого описується функцією від частоти $\mathbf{E}(v)$. Якщо хвильовий вектор $\boldsymbol{k}$ напрямлено перпендикулярно поверхні зразка, то вектори напруженості електромагнітного поля циркулярно поляризованої хвилі $\mathbf{E}(v)$ та $\mathbf{E}^{\prime}(v)$ будуть, у свою чергу паралельні поверхні зразка, а їх векторний добуток є пропорційним по відношенню до значення густини фотоструму $\boldsymbol{I}$, що виникає у зразку, а також визначає напрям відповідного вектору (рис. 1).

Модель генерації магнонного спінового фотоструму у зразку антиферомагнітного діелектрику може бути побудована аналогічним чином. У даному випадку математичний апарат має включати векторний добуток векторів магнітної індукції В × B' (рис. 2).

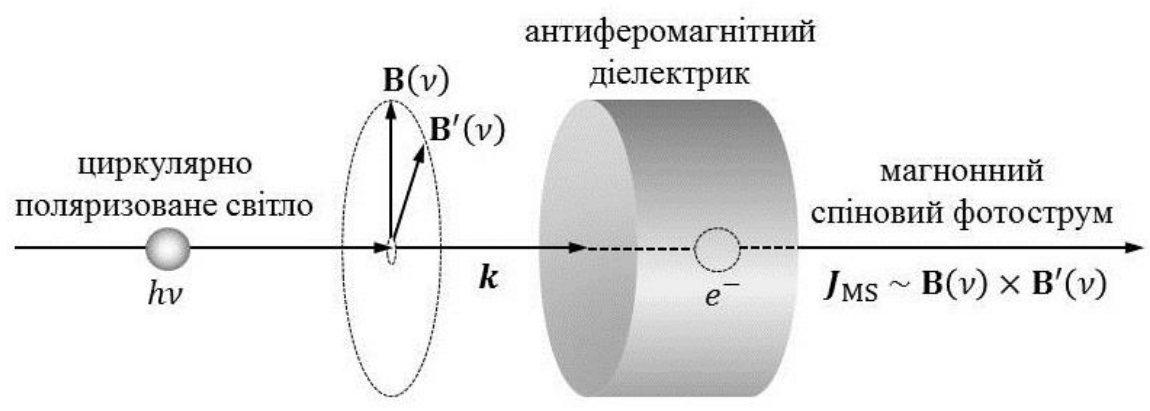

Рис. 2. Модель генерації магнонного спінового фотоструму циркулярно поляризованим світлом у зразку антиферомагнітного діелектрику 
На основі векторного добутку можна визначити електромагнітну хіральність зразка [13-15], як показник, що характеризує асиметрію у взаємодії світла з речовиною [16-18]. Характерно, що зміна величини магнітної складової оптичної хіральностіможе бути визначена через залежність $\chi^{\mathrm{M}}(k) \sim i \boldsymbol{k} \cdot \boldsymbol{B}(\boldsymbol{k}) \times \boldsymbol{B}^{\prime}(\boldsymbol{k})$. Нерівномірний розподіл сукупності магнонів можна розглядати як передачу спінового кутового моменту від світла до антиферомагнітних структур, що призводить до генерації спінового фотоструму, причому для такої системи можна визначити умови резонансу взаємодії.

\section{2. Моделювання процесу генерації магнонного спінового фотоструму}

Моделювання оптичної генерації спінових струмів може бути проведено на базі класичної електродинамічної моделі. Так, наприклад, для антиферомагнетика 3 кубічної кристалічною структурою можна виділити дві підрешітки $\boldsymbol{M}_{1} \mathrm{i} \boldsymbol{M}_{2}$, на основі чого можна визначити густину енергії через введення коефіцієнтів обміну між підрешітками $c_{1}$ i $c_{2}$ та коефіцієнт легко осьової анізотропії $c_{3}$ Вздовж вісі $z$ :

$W=c_{1} \cdot \boldsymbol{M}_{1} \cdot \boldsymbol{M}^{*}+c_{2} \cdot \nabla \boldsymbol{M}_{1} \cdot \nabla \boldsymbol{M}^{*}-c_{3} \cdot\left(\left(\boldsymbol{M}_{1}^{z}\right)^{2}+\left(\boldsymbol{M}_{2}^{z}\right)^{2}\right)$.

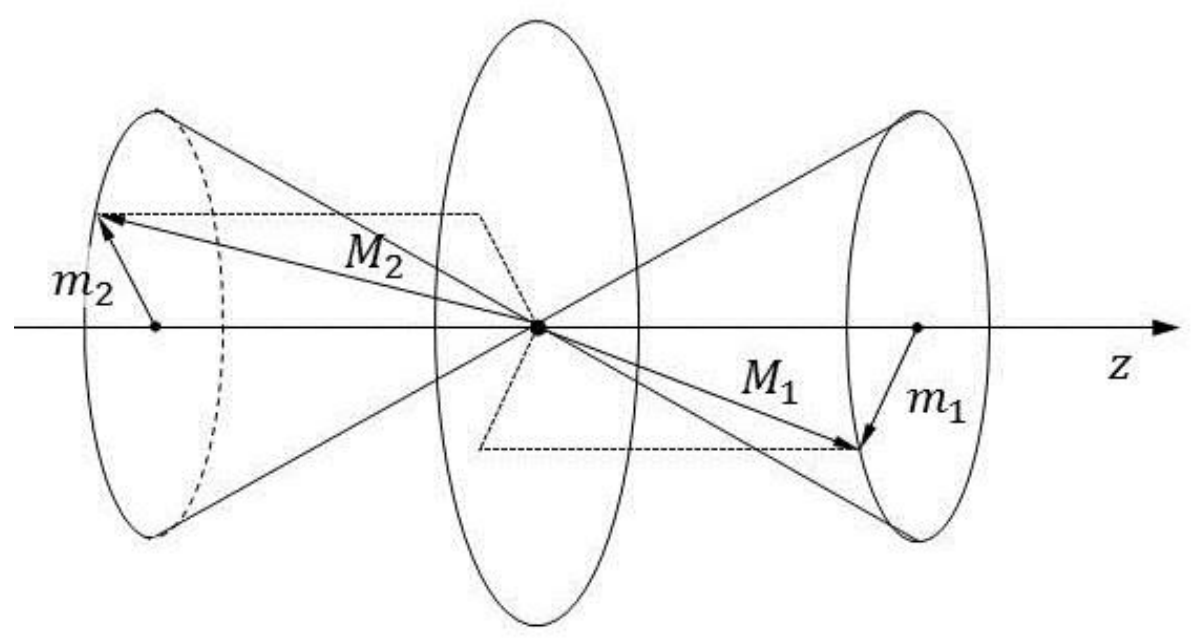

Рис. 3. Схема прецесійного руху векторів магнетизаиії підрешіток антиферомагнетиказ кубічної кристалічною структурою

На базі лінеаризованого рівняння Ландау-Лівшица можна записати наступну систему рівнянь для флуктуаційних компонент підрешітки:

$$
\left\{\begin{array}{l}
\partial_{t} p_{k}=-\gamma \cdot M_{s} \cdot\left(2 c_{3}-c_{2} \cdot k^{2}\right) \cdot z_{u} \times q_{k} \\
\partial_{t} q_{k}=-\gamma \cdot M_{s} \cdot\left(2 c_{1}+c_{2} \cdot k^{2}+2 c_{3}\right) \cdot z_{u} \times p_{k}
\end{array},\right.
$$

$$
\left\{\begin{array}{l}
p_{k}=m_{1 k}+m_{2 k} \\
q_{k}=m_{1 k}-m_{2 k}
\end{array}\right.
$$

На основі зазначеної системи рівнянь можна вивести наступні складові математичного апарату моделі генерації магнонного спінового фотоструму у середовищі антиферомагнітного діелектрику:

- магнетизація насичення $M_{s}$;

- компоненти Фур’є-розкладу функціїзбудження магнетизації $m_{1 k}$ відносно впорядкованого стану, що може бути описано ;

- одиничний вектор вздовж вісі $z$;

- гіромагнітне відношення $\gamma$.

При цьому впорядкований стан системи описується через наступну рівність: 
$M_{i}=m_{i}+(-1)^{i+1} \cdot M_{s} \cdot z_{u}$.

Враховуючи те, що обертальна симетрія зберігається при взаємодії між електромагнітним полем і системою спінів, вираз для спінового струму як функції від аргументів $p_{k}$ i $q_{k}$ можна знайти 3 рівняння безперервності для $z$-складової щільності намагніченості:

$m^{z}=\frac{m_{2}^{2}-m_{1}^{2}}{2 \cdot M_{s}}$

На основі проведеного аналізу розраховується величина щільності магнонного спінового фотоструму:

$I_{s}=-i \cdot \frac{\sum_{k}\left(\frac{\partial\left(2 c_{\mathbb{B}}-c_{\mathrm{Z}} k^{2}\right)}{\partial k}\left(p_{k}^{*} \times p_{k}\right)_{z}+\frac{\partial\left(2 c_{1}+c_{\mathrm{z}} k^{2}+2 c_{\mathrm{Z}}\right)}{\partial k}\left(q_{k}^{*} \times q_{k}\right)_{z}\right)}{4 \cdot M_{S}}(7)$

Таким чином, динаміка зміни намагніченості, збуджена електромагнітною хвилею світла, котрим опромінюється зразок, пов'язана 3 напрямом вісі $z$ i, відповідно, значення $\boldsymbol{p}_{\boldsymbol{k}} \mathrm{i}$ $\boldsymbol{q}_{\boldsymbol{k}}$ пропорційні $\boldsymbol{B}(\boldsymbol{k})$.

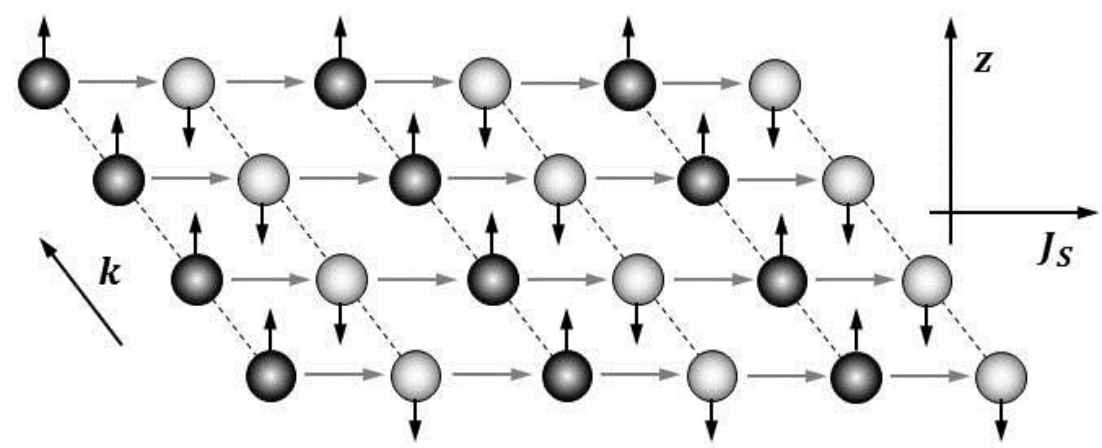

Рис. 4. Схема збудження спінового фотоструму у середовищі антиферомагнетика з кубічною кристалічною структурою

Запропонована математична модель відображає збудження спінового фотоструму у середовищі антиферомагнетика 3 кубічною кристалічною структурою, що представлена на рис. 4, де чорні стрілки відображають спіни, а сірі - антисиметричний обмін. Але математичний апарат також може бути адаптовано для відображення процесу збудження спінового фотоструму у середовищі антиферомагнетика 3 гексагональною кристалічною структурою, що $є$ актуальним для багатьох наноструктурних матеріалів на базі антиферомагнітних діелектриків.

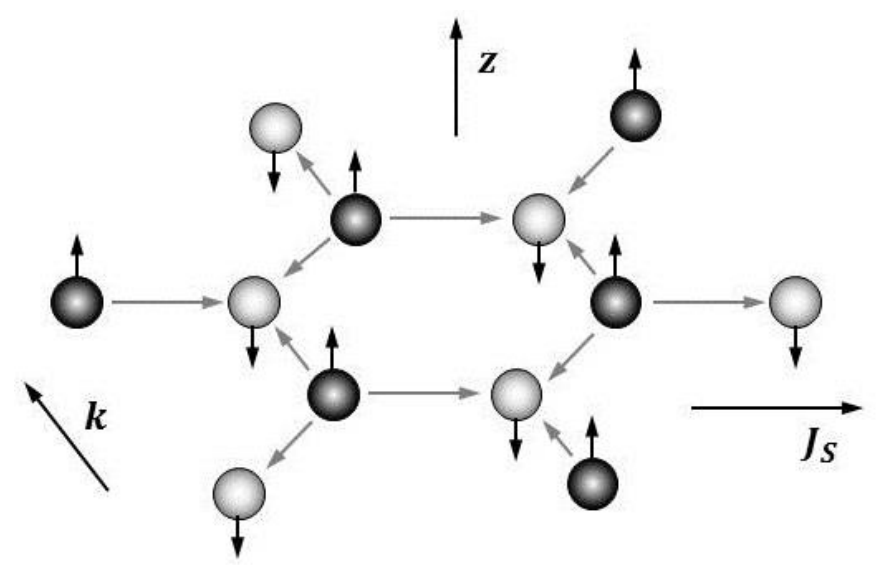

Рис. 5. Схема збудження спінового фотоструму у середовищі антиферомагнетика з гексагональною кристалічною структурою 
Відповідно, розроблена методологічна база може бути надалі використана дляаналізу процесів генерації та циркуляції спінових магнонних струмів у відповідності до структурних особливостей середовища антиферомагнітних діелектриків.

Висновки. В результаті аналізу базових фізичних принципів, що лежать у основі кругового фотогальванічного ефекту визначено особливості виникнення магнонного спінового фотоструму у структурах антиферомагнітних діелектриків, зазначено його нелінійність та запропоновано області його використання у пристроях опто-спінтроніки. При побудовіматематичноїмоделі розгляд спінового імпульсу було перенесено з фотонів на магнони. Показано, що циркулярно поляризоване електромагнітне поле в рамках моделі генерує прямий спіновий фотострум магнона, причому напрям струму визначається спіральністю світла. Розроблена математична модель була вдосконалена через врахування внеску геометричної організації складних гратчастих структур антиферомагнітних діелектриків і явище антисиметричного обміну.

\section{References}

1. Satoh, T., Iida, R., Higuchi, T., Fujiii, Y., Koreeda, A., Ueda, H., ... \& Ivanov, B. A. (2017). Excitation of coupled spin-orbit dynamics in cobalt oxide by femtosecond laser pulses. Nature communications, 8(1), 1-6.

2. Němec, P., Fiebig, M., Kampfrath, T., \& Kimel, A. V. (2018). Antiferromagnetic opto-spintronics. Nature Physics, 14(3), 229241.

3. Valev, V. K., Govorov, A. O., \& Pendry, J. (2017). Chirality and Nanophotonics: Chirality and Nanophotonics (Advanced Optical Materials 16/2017). Advanced Optical Materials, 5(16).

4. Tang, Y., \& Cohen, A. E. (2011). Enhanced enantioselectivity in excitation of chiral molecules by superchiral light. Science, 332(6027), 333-336.

5. Lovesey, S. W., Collins, J. T., \& Collins, S. P. (2019). Superchiral photons unveil magnetic circular dichroism. Physical Review $B, 99(5), 054428$.

6. Bliokh, K. Y., Kivshar, Y. S., \& Nori, F. (2014). Magnetoelectric effects in local light-matter interactions. Physical review letters, 113(3), 033601.

7. Canaguier-Durand, A., Hutchison, J. A., Genet, C., \& Ebbesen, T. W. (2013). Mechanical separation of chiral dipoles by chiral light. New Journal of Physics, 15(12), 123037.

8. Starosta, K., Koike, T., Chiara, C. J., Fossan, D. B., \& Vaman, C. (2002, April). Chirality and angular momentum coupling in odd-odd nuclei. In AIP Conference Proceedings (Vol. 610, No. 1, pp. 815-819). American Institute of Physics.

9. Lüth, H., \& Ibach, H. (2003). Solid-state physics: an introduction to principles of materials science. Springer-Verlag Berlin Heidelberg.

10. Proskurin, I., Ovchinnikov, A. S., Kishine, J. I., \& Stamps, R. L. (2018). Excitation of magnon spin photocurrents in antiferromagnetic insulators. Physical Review B, 98(13), 134422.

11. Sharma, S., Blanter, Y. M., \& Bauer, G. E. (2017). Light scattering by magnons in whispering gallery mode cavities. Physical Review B, 96(9), 094412.

12. Johansen, Ø., \& Brataas, A. (2018). Nonlocal coupling between antiferromagnets and ferromagnets in cavities. Physical review letters, 121(8), 087204.

13. Nolting, W., \& Ramakanth, A. (2009). Quantum theory of magnetism. Springer Science \& Business Media.

14. Lin, W., Chen, K., Zhang, S., \& Chien, C. L. (2016). Enhancement of thermally injected spin current through an antiferromagnetic insulator. Physical review letters, 116(18), 186601.

15. Takei, S., Moriyama, T., Ono, T., \& Tserkovnyak, Y. (2015). Antiferromagnet-mediated spin transfer between a metal and a ferromagnet. Physical Review B, 92(2), 020409.

16. Khymyn, R., Lisenkov, I., Tiberkevich, V. S., Slavin, A. N., \& Ivanov, B. A. (2016). Transformation of spin current by antiferromagnetic insulators. Physical Review B, 93(22), 224421.

17. Hoffmann, M. C., Khanna, V., \& Cavalleri, A. (2010). Noncollinear Broadband Terahertz-pump-Terahertz-probe spectroscopy of semiconductors. International Conference on Ultrafast Phenomena. doi: 10.1364/up.2010.me42.

18. Hirori, H., Doi, A., Blanchard, F., \& Tanaka, K. (2011). Single-cycle terahertz pulses with amplitudes exceeding $1 \mathrm{MV} / \mathrm{cm}$ generated by optical rectification in LiNbO 3. Applied Physics Letters, 98(9), 091106.

19. Kawano, M., \& Hotta, C. (2019). Thermal Hall effect and topological edge states in a square-lattice antiferromagnet. Physical Review B, 99(5), 054422.

20. Okuma, N. (2018). Quantum Theory of Antiferromagnetic Opto-spintronics: Reciprocal and Nonreciprocal Magnons Coupled with Polarized Photons. arXiv preprint arXiv:1805.08226.

21. Owerre, S. A., Mellado, P., \& Baskaran, G. (2019). Photoinduced Floquet topological magnons in Kitaev magnets. EPL (Europhysics Letters), 126(2), 27002.

22. Matusiak, M., Babij, M., Pomjakushina, E., \& Conder, K. (2016). Wiedemann-Franz law in iron-based superconductor Fe1+ dTe1- xSex. physica status solidi (b), 253(8), 1607-1611.

23. Kimura, T., Hamrle, J., Yang, T., \& Otani, Y. (2005, April). Magnetization switching due to non-local spin injection into small ferromagnetic particle. In 2005 IEEE International Magnetics Conference (INTERMAG) (pp. 133-134). IEEE.

24. Okubo, S., Ueda, T., Ohta, H., Zhang, W., Sakurai, T., Onishi, N., ... \& Sakai, T. (2012). Dzyaloshinsky-Moriya interaction and field-induced magnetic order in an antiferromagnetic honeycomb lattice compound Bi 3 Mn 4 O 12 (NO 3) studied by high-field electron spin resonance. Physical Review B, 86(14), 140401. 